\title{
WHAT IS THE HIGHER-DIMENSIONAL INFINITESIMAL GROUPOID OF A MANIFOLD?
}

\author{
DENNIS BORISOV
}

(Received 30 July 2010; accepted 21 March 2011)

Communicated by S. Rosenberg

Dedicated to Alan Carey, on the occasion of his 60th birthday

\begin{abstract}
The construction (by Kapranov) of the space of infinitesimal paths on a manifold is extended to include higher-dimensional infinitesimal objects, encoding contractions of infinitesimal loops. This full infinitesimal groupoid is shown to have the algebra of polyvector fields as its nonlinear cohomology.
\end{abstract}

2010 Mathematics subject classification: primary 22A22; secondary 18D05.

Keywords and phrases: Lie algebroid, Lie-Rinehart algebras, higher categories, polyvector fields.

\section{Introduction}

What is the infinitesimal version of the fundamental groupoid of a manifold $\mathcal{M}$ ? The standard answer is that it is the Lie-Rinehart algebra (also called the Lie algebroid) of vector fields on $\mathcal{M}$. However, this answer is not precise.

Let $\alpha$ and $\beta$ be two vector fields on $\mathcal{M}$. Assume for simplicity that they commute, that is, $[\alpha, \beta]=0$. The left-hand side of this equation represents a loop, while the righthand side stands for the constant path. By equating the two sides we actually contract the loop.

In [2] another Lie-Rinehart algebra was constructed for each $\mathcal{M}$. Starting with the space $\mathfrak{X}^{1}$ of vector fields, one builds the free Lie-Rinehart algebra $\mathcal{R}\left(\mathfrak{X}^{1}\right)$, generated by $\mathfrak{X}^{1}$. The action of $\mathcal{R}\left(\mathfrak{X}^{1}\right)$ on functions on $\mathcal{M}$ is generated by the action of $\mathfrak{X}^{1}$, but otherwise the Lie bracket is free. Because the Lie bracket is free, one does not contract nondegenerate loops (we still have $\llbracket \alpha, \alpha \rrbracket=\llbracket \alpha, 0 \rrbracket=0$ ) and therefore one can call $\mathcal{R}\left(\mathfrak{X}^{1}\right)$ the space of infinitesimal paths. This is a module over the algebra $\mathfrak{X}^{0}$ of functions on $\mathcal{M}$ and it defines a vector bundle on $\mathcal{M}$ that we will denote by $R T M$. Unless $\operatorname{dim}(\mathcal{M}) \leq 1$, the vector bundle $\mathcal{R} T \mathcal{M}$ is obviously infinite-dimensional.

While $\mathfrak{X}^{1}$ is obtained by contracting all loops, $\mathcal{R}\left(\mathfrak{X}^{1}\right)$ is built by avoiding contractions. In this paper we add higher-dimensional components to $\mathcal{R}\left(\mathfrak{X}^{1}\right)$ that represent the higher-dimensional submanifolds which are needed to parametrize

(c) 2011 Australian Mathematical Publishing Association Inc. 1446-7887/2011 \$16.00 
contractions. We obtain the full infinitesimal groupoid $\mathbb{X}^{*}$, graded by the dimension of submanifolds. The algebraic structure on $\mathbb{X}^{*}$ is considerably more complicated than that of a Lie-Rinehart algebra. In particular, there are the homotopy maps

$$
\mathbb{X}^{k} \rightarrow \mathbb{X}^{k-1}
$$

that represent contractions of loops. For example, $\mathbb{X}^{1}=\mathcal{R}\left(\mathfrak{X}^{1}\right)$, but $\mathbb{X}^{1}$ modulo the image of $\mathbb{X}^{2} \rightarrow \mathbb{X}^{1}$ is just $\mathfrak{X}^{1}$.

We also consider the 'nonlinear cohomology' $\mathcal{H}\left(\mathbb{X}^{*}\right)$ of $\mathbb{X}^{*}$ (for $k>1$ the set $\mathbb{X}^{k}$ is not additive). This is the homotopy classes of elements of $\mathbb{X}^{*}$, that do not themselves define nontrivial equivalence relations. In this case, $\mathcal{H}\left(\mathbb{X}^{*}\right)$ is the algebra of polyvector fields $\sum_{k=1}^{-\infty} \bigwedge^{-k+1} \mathfrak{X}^{1}$. It is important to note that this algebra is not Gerstenhaber since the Lie bracket has degree zero, while the wedge product is of degree -1 . Here we use the cohomological notation, that is, differentials raise degrees.

The idea of the construction of $\mathbb{X}^{*}$ is as follows. A loop like $[\alpha, \beta]$ is contracted by a 2-morphism, that is, it occurs inside a jet of a submanifold of dimension two. While $\alpha$ and $\beta$ are given as 1 -jets, it is not enough to take the 1 -jet of the surface. Indeed, if $\alpha$ and $\beta$ are given as morphisms

$$
\alpha: \mathfrak{X}^{0} \rightarrow \mathfrak{X}^{0} \otimes \mathbb{R}\left[\epsilon_{1}\right] /\left(\epsilon_{1}^{2}\right), \quad \beta: \mathfrak{X}^{0} \rightarrow \mathfrak{X}^{0} \otimes \mathbb{R}\left[\epsilon_{2}\right] /\left(\epsilon_{2}^{2}\right),
$$

then their bracket requires $\epsilon_{1} \epsilon_{2}$ and hence we need a morphism

$$
\mathfrak{X}^{0} \rightarrow \mathfrak{X}^{0} \otimes \mathbb{R}\left[\epsilon_{1}, \epsilon_{2}\right] /\left(\epsilon_{1}^{2}, \epsilon_{2}^{2}\right) .
$$

In other words, we need to consider sections of the second tangent bundle $T^{2} \mathcal{M}$. If $v \in \mathfrak{X}^{2}$ is such a section, then it has $\alpha$ and $\beta$ as its two projections to $\mathfrak{X}^{1}$. In addition, it defines a homotopy relation in $\mathbb{X}^{1}$ by equating $[\alpha, \beta]$ and $\llbracket \alpha, \beta \rrbracket$, where $\llbracket-,-\rrbracket$ is the free bracket on $\mathbb{X}^{1}=\mathcal{R}\left(\mathfrak{X}^{1}\right)$.

To understand how $v$ provides the identification $[\alpha, \beta]=\llbracket \alpha, \beta \rrbracket$, one should note that there are two ways to view $v$ as a one-parameter family of vector fields on $\mathcal{M}$. On the one hand $v$ is tangent to $\alpha$, while on the other it is tangent to $\beta$. Choosing one of the ways is equivalent to choosing an order on the pair $\{\alpha, \beta\}$, that is, choosing an orientation on $v$. The symmetric group $\mathbb{S}_{2}$ acts on the set of these choices, and if $\sigma \in \mathbb{S}_{2}$ is the nontrivial element, then its action is well known to produce $[\alpha, \beta]$. Indeed, let $\alpha_{*}(\beta)$ and $\beta_{*}(\alpha)$ be the sections of $T^{2} \mathcal{M}$ obtained by using functoriality of $T$. Then

$$
\alpha_{*}(\beta)-\beta_{*}(\alpha)=[\alpha, \beta],
$$

where we use the strong difference of points in $T^{2} \mathcal{M}$ on the left-hand side (see $\left.[3,5]\right)$ and we use the identification of points in $T \mathcal{M}$ with tangents to the fibers of $T M \rightarrow \mathcal{M}$ on the right-hand side. Having two brackets $[-,-]$ and $\llbracket-,-\rrbracket$ on $\mathcal{R} T M$ gives two actions of $\mathbb{S}_{2}$ on $T(\mathcal{R} T \mathcal{M})$. Taking their strong difference, we obtain $[\alpha, \beta]-\llbracket \alpha, \beta \rrbracket$.

There are many sections of $T^{2} \mathcal{M}$ that have $\alpha$ and $\beta$ as their projections onto $\mathfrak{X}^{1}$. Therefore there are many different ways to contract the loop $[\alpha, \beta]$. In order to equate these different ways, we need to consider jets of submanifolds of dimension three. Again these jets should be 3 -jets of a particular kind, that is, maps to $\mathbb{R}\left[\epsilon_{1}, \epsilon_{2}, \epsilon_{3}\right] /\left(\epsilon_{i}^{2}\right)$. 
So we need sections of $T^{3} \mathcal{M}$. Since the combinatorics of $\left\{T^{k} \mathcal{M}\right\}$ are not globular, but cubical, a section $\mu: \mathcal{M} \rightarrow T^{3} \mathcal{M}$ defines several homotopy relations on $\mathbb{X}^{2}$. There are three pairs of generators in $\mathbb{R}\left[\epsilon_{1}, \epsilon_{2}, \epsilon_{3}\right] /\left(\epsilon_{i}^{2}\right)$ and hence there are three homotopy maps $\mathfrak{X}^{3} \rightarrow \mathbb{X}^{2}$. For $k$ chosen arbitrarily, $v \in \mathfrak{X}^{k}$ defines $k ! /[2(k-2) !]$ homotopies.

To continue this construction further we have to work with iterations of $\mathcal{R} T$ rather than with iterations of the usual tangent bundle. That is, instead of $T^{2} \mathcal{M}$, we should take sections in $(\mathcal{R} T)^{2} \mathcal{M}$. One can construct $(\mathcal{R} T)^{2} \mathcal{M}$, but it is too big. It contains infinitesimal loops that are completely inside the fibers of $\mathcal{R} T \mathcal{M} \rightarrow \mathcal{M}$. Tangents to these fibers represent infinitesimal automorphisms of tangents to $\mathcal{M}$ and, as far as $\mathcal{M}$ is concerned, infinitesimal loops in these fibers should be contracted.

This leads us to the construction of relatively free Lie-Rinehart algebras. We formulate this in general terms. Let $\pi: \mathcal{N}_{1} \rightarrow \mathcal{N}_{2}$ be a smooth map that is locally (on $\mathcal{N}_{1}$ ) a trivial bundle, but not necessarily linear. We define $\mathcal{R}\left(\mathfrak{X}^{1}\left(\mathcal{N}_{1}\right), \pi\right)$ to be the space of infinitesimal paths, obtained from $\mathcal{R}\left(\mathfrak{X}^{1}\left(\mathcal{N}_{1}\right)\right)$ by contracting all 'vertical loops' with respect to $\pi$. If $\pi: \mathcal{N}_{1} \rightarrow \mathrm{pt}$ is the unique map to a point, then we obtain the usual space of vector fields. If $\pi: \mathcal{N}_{1} \rightarrow \mathcal{N}_{1}$ is the identity map, then we obtain the space $\mathcal{R}\left(\mathfrak{X}^{1}\left(\mathcal{N}_{1}\right)\right)$ of all infinitesimal paths from [2].

Applying this construction to $\mathcal{M}$ and iterating, we obtain a sequence $\left\{\mathbb{T}^{k} \mathcal{M}\right\}$ which, just like $\left\{T^{k} \mathcal{M}\right\}$, is a semisimplicial diagram of linear bundles. We define $\mathbb{X}^{k}$ to be the set of sections $\mathcal{M} \rightarrow \mathbb{T}^{k} \mathcal{M}$.

For $k>1$ the set $\mathbb{X}^{k}$ is not additive, but it has $k$ different additions over $\mathbb{X}^{k-1}$. Also, there is a cup product and a composition product, encoding infinitesimal automorphisms and Lie derivatives, respectively. Taking nonlinear cohomology as described above and factoring out jets of degenerate submanifolds, we obtain the algebra of polyvector fields.

We now describe the organization of this paper. In Section 2 we recall the construction and some algebraic properties of the usual iterated tangent bundles. We describe the additional structure one has on the sets of sections. In Section 3 we give the construction of relatively free Lie-Rinehart algebras and discuss their functorial properties. In Section 4 we construct the full infinitesimal groupoid $\mathbb{X}^{*}$, discuss some of the algebraic operations defined on this groupoid, including actions of symmetric groups, and compute its cohomology $\mathcal{H}\left(\mathbb{X}^{*}\right)$.

Everything in this paper is formulated for smooth real manifolds. All of the statements and proofs are also valid if one uses complex analytic manifolds instead.

\section{2. $k$-vectors and $k$-vector fields}

In this section we recall the basic properties of points and sections of iterated tangent bundles. In particular, we describe decompositions of sections into sets of vector fields, subject to the action by symmetric groups. These decompositions, and the action, will be central to our treatment of full infinitesimal groupoids in Section 4.

Let $\mathcal{M}$ be a smooth manifold of dimension $n$. Let $T^{k} \mathcal{M}$ for $k \geq 0$ be its $k$ th iterated tangent bundle. That is, $T^{k} \mathcal{M}$ is the tangent bundle on $T^{k-1} \mathcal{M}$ and $T^{0} \mathcal{M}=\mathcal{M}$. It is 
well known (see, for example, [1, 6]) that for each $k \geq 1$ there are $k$ vector-bundle projections

$$
\left\{\pi_{k, i}: T^{k} \mathcal{M} \rightarrow T^{k-1} \mathcal{M}\right\}_{0 \leq i \leq k-1}
$$

where $\left\{\pi_{k, i}\right\}_{k \geq 1}$ satisfy the usual equations of simplicial boundaries. From the (semi)simplicial properties of $\left\{\pi_{k, i}\right\}_{k \geq 1}$ and the equality

$$
\pi_{1} \circ \pi_{2,0}=\pi_{1} \circ \pi_{2,1},
$$

it follows easily that for any $k \geq 1$ all possible projections $T^{k} \mathcal{M} \rightarrow \mathcal{M}$ are equal. We will denote by $\mathfrak{X}^{k}$ the set of smooth sections $\mathcal{M} \rightarrow T^{k} \mathcal{M}$. We will call such sections $k$-vector fields. The evaluations of these $k$-vector fields at points of $\mathcal{M}$ will be called $k$-vectors.

There are different ways to interpret points in $T^{k} \mathcal{M}$. We will use the notion of $F$-equivalence introduced in [6] since it is very well suited to the treatment of $k$-morphisms, defined as jets of $k$-dimensional submanifolds in $\mathcal{M}$. Let

$$
v, v^{\prime}:\left(\mathbb{R}^{k}, 0\right) \rightarrow(\mathcal{M}, p)
$$

be two $k$-jets. We say that $v$ is $F$-equivalent to $v^{\prime}$ if, for any function $f$ on $\mathcal{M}$ around $p$ and any $1 \leq m \leq k$,

$$
\left.\frac{\partial^{m}}{\partial_{i_{1}} \cdots \partial_{i_{m}}}(f \circ v)\right|_{0}=\left.\frac{\partial^{m}}{\partial_{i_{1}} \cdots \partial_{i_{m}}}\left(f \circ v^{\prime}\right)\right|_{0},
$$

where the $i_{j}$ are pairwise different. Obviously this equivalence relation depends on the choice of the coordinate system for $\mathbb{R}^{k}$.

Proposition 2.1 (See $[1,6])$. Let $p$ be a point on $\mathcal{M}$. There is a bijective correspondence between $k$-vectors and $F$-equivalence classes of $k$-jets of maps $\left(\mathbb{R}^{k}, 0\right) \rightarrow(\mathcal{M}, p)$. This correspondence is natural in $\mathcal{M}$.

The semisimplicial structure on $\left\{T^{k} \mathcal{M}\right\}_{k \geq 1}$ corresponds to the diagram of coordinate subspaces of $\mathbb{R}^{n}$. More precisely, let $\left\{x_{i}\right\}_{0 \leq i<k}$ be the natural coordinate system on $\mathbb{R}^{k}$. Then each $v:\left(\mathbb{R}^{k}, 0\right) \rightarrow(\mathcal{M}, p)$ has $k$ faces:

$$
v_{i}:\left(\mathbb{R}^{k-1}, 0\right) \rightarrow(\mathcal{M}, p), \quad 0 \leq i \leq k-1,
$$

with $v_{i}$ being the restriction of $v$ to the linear subspace of $\mathbb{R}^{k}$ given by the vanishing of $x_{i}$. If we choose a simplicial model for the $n$-groupoid of $\mathcal{M}$, that is, if we consider $k$-simplices in $\mathcal{M}$ (submanifolds with corners) as $k$-morphisms, then it is clear that we can realize $v$ as the jet of a $k$-morphism between jets of $(k-1)$ morphisms $\left\{v_{0}, \ldots, v_{k-1}\right\}$. Note that $k$-morphisms are represented by $k$-jets, while $(k-1)$-morphisms are represented by $(k-1)$-jets. This is not really an inconsistency since, for a $(k-1)$-dimensional submanifold of $\mathcal{M}$, the $F$-equivalence class of its $k$-jet is completely determined by its $(k-1)$-jet.

To describe reparametrizations of $k$-vectors and $k$-fields, that is, suitable changes of coordinates on $\mathbb{R}^{k}$, we use the dual language of morphisms between algebras of functions. Consider a sequence of Weil algebras

$$
\mathcal{W}_{k}:=\mathbb{R}\left[\epsilon_{0}, \ldots, \epsilon_{k-1}\right] /\left\{\epsilon_{i}^{2}\right\}_{0 \leq i \leq k-1}, \quad k \geq 1 .
$$


For any point $p \in \mathcal{M}$, let $\mathfrak{X}_{p}^{0}$ be the stalk at $p$ of the sheaf of functions on $\mathcal{M}$. Then points in $T^{k} \mathcal{M}$ over $p$ correspond to morphisms of $\mathbb{R}$-algebras

$$
\mathfrak{X}_{p}^{0} \rightarrow \mathcal{W}_{k}
$$

that factor the evaluation map $\mathfrak{X}_{p}^{0} \rightarrow \mathbb{R}$. Automorphisms of $W_{k}$ provide reparametrizations of $k$-vectors and lead to polynomial groups [1].

Amongst all of the automorphisms, we will be particularly interested in the action of the symmetric group $\mathbb{S}_{k}$ which permutes generators of $\mathcal{W}_{k}$. These permutations induce an action of $\mathbb{S}_{k}$ on $T^{k} \mathcal{M}$, and this action is important to us since choosing the order on the generators of $\mathcal{W}_{k}$ allows us to decompose any section $\mathcal{M} \rightarrow T^{k} \mathcal{M}$ into a set of sections $\mathcal{M} \rightarrow T \mathcal{M}$. This decomposition will allow us to introduce several important operations on the sections of $T^{k} \mathcal{M}$, and these operations are well defined since they are $\mathbb{S}_{k}$-invariant.

It is well known (see, for example, [1]) that any $k$-vector field $v \in \mathfrak{X}^{k}$ can be decomposed into a set $\left\{\alpha_{\phi}\right\}$ of 1-vector fields, indexed by the nonempty subsets $\phi \subseteq\{0, \ldots, k-1\}$. Since there are several different possible ways to decompose a $k$-vector field, we discuss these in detail here. We start with an example of $\beta \in \mathfrak{X}^{2}$.

The two projections $T^{2} \mathcal{M} \rightrightarrows T \mathcal{M}$ map $\beta$ to two 1 -vector fields $\alpha_{0}$ and $\alpha_{1}$. The image of $\alpha_{0}: \mathcal{M} \rightarrow T \mathcal{M}$ is transversal to the fibers of $T \mathcal{M}$ over $\mathcal{M}$ and hence we have a decomposition of the tangent spaces into vertical and horizontal parts at the points of the image. Applying this decomposition to $\beta$, we obtain

$$
\beta \mapsto\left\{\alpha_{0}, \alpha_{1}, \alpha_{01}\right\}, \quad \alpha_{0}, \alpha_{1}, \alpha_{01} \in \mathfrak{X}^{1},
$$

where $\alpha_{01}$ is tangent to the fibers of $T \mathcal{M} \rightarrow \mathcal{M}$. This decomposition, however, depends on the choice of $\alpha_{0}$ as the first projection.

More precisely, there is the canonical action of the symmetric group $\mathbb{S}_{2}$ on $T^{2} \mathcal{M}$ and of course we can permute $\alpha_{0}$ and $\alpha_{1}$ in (2.1). If $\sigma \in \mathbb{S}_{2}$ is the nontrivial element,

$$
\sigma(\beta) \mapsto\left\{\alpha_{1}, \alpha_{0}, \alpha_{01}+\left[\alpha_{0}, \alpha_{1}\right]\right\}
$$

The appearance of $\left[\alpha_{0}, \alpha_{1}\right]$ in (2.2) is due to the following. Every function $f$ on $\mathcal{M}$ defines two functions on $T \mathcal{M}$ : one is composition with the projection $\pi_{1}: T \mathcal{M} \rightarrow \mathcal{M}$ and the other is $d f$. Reflecting this fact, we can combine the two lifts into one total lift $f \circ \pi_{1}+\epsilon d f$ on $T M$, where $\epsilon^{2}=0$. Since it is a collection of tangents to TM, the 2-vector field $\beta$ acts on the total lift $f \circ \pi_{1}+\epsilon d f$. Using the decomposition into horizontal and vertical parts provided by $\alpha_{0}$, we can write this action as follows:

$$
f \circ \pi_{1}+\epsilon d f \mapsto \alpha_{1}(f) \circ \pi_{1}+\epsilon\left(\alpha_{01}(f)+\alpha_{1} \alpha_{0}(f)\right) \circ \pi_{1} .
$$

In fact, $\beta$ is determined by its first projection to $T \mathcal{M}$, that is, $\alpha_{0}$ and its action (2.3) on the total lifts of functions from $\mathcal{M}$. The action on the $\epsilon$-part is that of a second-order differential operator and, given some choices, such as the order on the pair $\alpha_{0}, \alpha_{1}$, we can extract the first-order part, $\alpha_{01}$. The opposite choice produces a different extraction, $\alpha_{01}+\left[\alpha_{0}, \alpha_{1}\right]$. 
In general, a section $v: \mathcal{M} \rightarrow T^{k} \mathcal{M}$ is uniquely determined by $2^{k}-1$ sections $\left\{\alpha_{\phi}: \mathcal{M} \rightarrow T \mathcal{M}\right\}$, if we fix an order on $\phi$ s. We use the lexicographical order. Then a set $\left\{\alpha_{\phi}\right\}$ of 1 -vector fields uniquely determines a $k$-vector field. For each $\phi$ of size $m$, the corresponding differential operator of order $m$ is

$$
\sum_{\phi_{i}>\cdots>\phi_{1}} \alpha_{\phi_{i}} \circ \cdots \circ \alpha_{\phi_{1}},
$$

where the sum is taken over all decompositions $\phi=\bigcup_{1 \leq j \leq i} \phi_{j}$ into pairwise disjoint subsets.

The action of the symmetric group $\mathbb{S}_{k}$ on $T^{k} \mathcal{M}$ is expressed as follows. Let $0 \leq i<k-1$ and let $\sigma_{i, i+1} \in \mathbb{S}_{k}$ be the swapping of $i$ and $i+1$. Then $\sigma_{i, i+1}\left(v_{k}\right)$ is given by $\left\{\alpha_{\phi}^{\prime}\right\}$, where $\alpha_{\phi}^{\prime}=\alpha_{\psi}$, if $\sigma_{i, i+1}(\phi)=\psi$ and $\phi \neq \psi$. For the remaining nonempty subsets $\phi \subseteq\{0, \ldots, k-1\}$ we have

$$
\alpha_{\phi}^{\prime}=\alpha_{\phi}+\sum_{\phi^{\prime}<\phi^{\prime \prime}}\left[\alpha_{\phi^{\prime}}, \alpha_{\phi^{\prime \prime}}\right],
$$

where the sum is taken over all decompositions $\phi=\phi^{\prime} \cup \phi^{\prime \prime}$ such that $\phi^{\prime} \cap \phi^{\prime \prime}=\emptyset$ and $\sigma_{i, i+1}\left(\phi^{\prime}\right)>\sigma_{i, i+1}\left(\phi^{\prime \prime}\right)$.

Some operations on $k$-vector fields are performed pointwise, that is, they can also be defined for $k$-vectors. Other operations on $k$-vector fields require sections. Now we describe some of both types of these operations in terms of $k$-vector fields.

We have already mentioned that for each $k \geq 1$ there are $k$ vector bundle structures on $T^{k} \mathcal{M}$ over $T^{k-1} \mathcal{M}$. Obviously these $k$ additions on $T^{k} \mathcal{M}$ translate to $k$ additions on $\mathfrak{X}^{k}$. Two sections $\left\{\mu_{\phi}\right\}$ and $\left\{v_{\phi}\right\}$ can be added if there is a $(k-1)$-dimensional face $\psi \subset\{0, \ldots, k-1\}$, that is, $\psi$ has exactly $k-1$ elements such that

$$
\mu_{\phi}=v_{\phi} \quad \forall \phi \subseteq \psi .
$$

Then $\mu+_{\psi} v$ is given as follows. If $\phi \subseteq \psi$, then $\left(\mu+_{\psi} v\right)_{\phi}=\mu_{\phi}=v_{\phi}$. If $\phi \nsubseteq \psi$, then $\left(\mu+{ }_{\psi} v\right)_{\phi}=\mu_{\phi}+v_{\phi}$.

An operation that is closely related to the additions is the strong difference between two 2-vectors. It is not defined for every pair $\beta_{1}, \beta_{2} \in T^{2} \mathcal{M}$, but only for those that have same projections to $T M$, that is,

$$
\pi_{2,0}\left(\beta_{1}\right)=\pi_{2,0}\left(\beta_{2}\right), \quad \pi_{2,1}\left(\beta_{1}\right)=\pi_{2,1}\left(\beta_{2}\right) .
$$

In this case it is easy to see that the difference between $\beta_{1}$ and $\beta_{2}$ as vectors on $\pi_{2,0}\left(\beta_{1}\right) \in T \mathcal{M}$ is a vector tangent to the fiber of $\pi_{2,0}\left(\beta_{1}\right)$ over $\mathcal{M}$. This vector can be obtained by the vertical lift of some $\alpha \in T M$ which is, by definition, the strong difference of $\beta_{1}$ and $\beta_{2}$, that is,

$$
\alpha:=\beta_{1}-\beta_{2} .
$$

Clearly one can take the strong difference of a pair of $k$-vectors for any $k \geq 2$ which are considered as 2 -vectors on $T^{k-2} \mathcal{M}$. This operation is also defined for $k$ fields and, in terms of decompositions into 1-fields, it is represented as follows. Let $\mu=\left\{\alpha_{\phi}\right\}$ and $\nu=\left\{\beta_{\phi}\right\}$ be two $k$-fields. We can define $\mu-v$ only if all $(k-1)$-faces of $\mu$ 
and $v$ coincide, that is, for any $\phi \subset\{0, \ldots, k-1\}$ of size $\leq k-1$ we have $\alpha_{\phi}=\beta_{\phi}$. Then $\mu-v=\left\{\gamma_{\psi}\right\}$ is the $(k-1)$-field defined as follows: for any $\psi \subseteq\{0, \ldots, k-3\}$,

$$
\gamma_{\psi}=\alpha_{\psi}=\beta_{\psi}, \quad \gamma_{\psi \cup\{k-2\}}=\alpha_{\psi \cup\{k-2, k-1\}}-\beta_{\psi \cup\{k-2, k-1\}} .
$$

There is another important pointwise operation called the the cup product. However, it is only partially defined. Consider the Weil algebras

$$
\mathcal{V}_{k}:=\mathbb{R}\left[\varepsilon_{1}, \ldots, \varepsilon_{k}\right] /\left(\varepsilon_{i} \varepsilon_{j}\right) .
$$

For $k>1$ and $\mathcal{V}_{k} \neq \mathcal{W}_{k}$ there are several $\mathbb{R}$-algebra morphisms $\mathcal{V}_{k} \rightarrow \mathcal{W}_{k}$. Now let $p$ be a point in $\mathcal{M}$ and let $x: \mathfrak{X}_{p}^{0} \rightarrow \mathcal{W}_{k}$ and $y: \mathfrak{X}_{p}^{0} \rightarrow \mathcal{W}_{m}$ be a $k$-vector and an $m$-vector, respectively, at $p$. Suppose that $y$ factors through some $g: \mathcal{V}_{m} \rightarrow \mathcal{W}_{m}$. Then we can define a $k+m$-vector $x \cup y$ using the $\mathbb{R}$-algebra morphism

$$
\mathcal{W}_{k} \prod_{\mathbb{R}} \mathcal{V}_{m} \rightarrow \mathcal{W}_{k+m}, \quad \epsilon_{i} \mapsto \epsilon_{i}, \quad \varepsilon_{j} \mapsto h \circ g\left(\varepsilon_{j}\right) \epsilon_{0} \cdots \epsilon_{k-1},
$$

where $h: \mathcal{W}_{m} \rightarrow \mathcal{W}_{k+m}$ maps $\epsilon_{i}$ to $\epsilon_{k+i}$. For example, if $m=k=1$, then $x \cup y$ is the evaluation at $x$ of the well-known 'vertical lift' of $y$ to TM. Similarly, there are vertical lifts of 1 -vectors to $T^{k} \mathcal{M}$ for $k>1$. If $m>1$, then this operation is no longer everywhere defined. It is only defined for those $y: \mathfrak{X}_{p}^{0} \rightarrow \mathcal{W}_{m}$ that factor through $\mathcal{V}_{m}$.

Notice that the cup product is invariant with respect to the action of symmetric groups. That is, if $\sigma_{k} \in \mathbb{S}_{k}$ and $\sigma_{m} \in \mathbb{S}_{m}$, then

$$
\left(\sigma_{k} x\right) \cup\left(\sigma_{m} y\right)=\left(\sigma_{k} \times \sigma_{m}\right)(x \cup y) .
$$

In terms of sequences of 1-vector fields the cup product is represented as follows. Let $\left\{\alpha_{\phi}\right\}=\mu \in \mathfrak{X}^{k}$ and $\left\{\beta_{\psi}\right\}=v \in \mathfrak{X}^{m}$ and suppose that their cup product $\left\{\gamma_{\chi}\right\}=\mu \cup v \in \mathfrak{X}^{k+m}$ is everywhere defined (that is, $v: \mathfrak{X}^{0} \rightarrow \mathcal{W}_{m} \otimes_{\mathbb{R}} \mathfrak{X}^{0}$ factors through $\mathcal{V}_{m} \otimes_{\mathbb{R}} \mathfrak{X}^{0}$ ). Then

$$
\gamma_{\phi}=\alpha_{\phi}, \quad \gamma_{\phi \cup \psi}=\beta_{\psi},
$$

and the rest of the components are 0 . Here we consider the nonempty sets $\phi \subseteq$ $\{0, \ldots, k-1\}$ and $\psi \subseteq\{0, \ldots, m-1\}$ to be the subsets of $\{0, \ldots, k+m-1\}$ given by the (lexicographical) order preserving bijection

$$
\{0, \ldots, k-1\} \amalg\{0, \ldots, m-1\} \stackrel{\simeq}{\rightarrow}\{0, \ldots, k+m-1\} .
$$

An important operation that is not defined pointwise is the composition product of vector fields. Let $\mu \in \mathfrak{X}^{k}$ and $v \in \mathfrak{X}^{m}$. Since $\mu$ is a map $\mathcal{M} \rightarrow T^{k} \mathcal{M}$, applying the tangent functor $m$ times we obtain a map $\mu_{*}: T^{m} \mathcal{M} \rightarrow T^{m+k} \mathcal{M}$. Evaluating at $v: \mathcal{M} \rightarrow T^{m} \mathcal{M}$ we get a section $\mu \times v: \mathcal{M} \rightarrow T^{m+k} \mathcal{M}$. In terms of sequences of 1 -vector fields this operation is written as follows. Let $\left\{\alpha_{\phi}\right\},\left\{\beta_{\psi}\right\}$ and $\left\{\gamma_{\chi}\right\}$ be $\mu, v$ and $\mu \times v$, respectively. Then

$$
\gamma_{\phi}=\alpha_{\phi}, \quad \gamma_{\psi}=\beta_{\psi},
$$

and the rest of the components are 0 . Here we again consider $\phi$ and $\psi$ to be subsets of $\{1, \ldots, k+m\}$ using (2.5). Notice also that the composition product is invariant with 
respect to the action of symmetric groups, that is, for any $\sigma_{k} \in \mathbb{S}_{k}$ and $\sigma_{m} \in \mathbb{S}_{m}$ we have

$$
\left(\sigma_{k} \mu\right) \times\left(\sigma_{m} v\right)=\left(\sigma_{k} \times \sigma_{m}\right)(\mu \times v) .
$$

\section{Relatively free Lie-Rinehart algebras}

In this section we recall (from [2]) the construction of the bundle of infinitesimal paths on a manifold and relativize it. That is, we construct bundles of infinitesimal paths where we contract some of the loops. This relative version has nice functorial properties. We use these properties in the next section to build the iterated bundles of infinitesimal paths on a manifold.

In [2] the space of infinitesimal paths on $\mathcal{M}$ is defined to be the free Lie-Rinehart algebra, generated by $\mathfrak{X}^{1}$. Here are the details of this free construction in the general case. Let $A$ be an $\mathbb{R}$-algebra and let $M$ be an $A$-module with an anchor, that is, such that there is an $A$-map $M \rightarrow \operatorname{Der}_{\mathbb{R}}(A)$. Let $\mathcal{L}(M)$ be the free Lie algebra over $\mathbb{R}$ generated by $M$. By its construction, $\mathcal{L}(M)$ is a graded space with $\mathcal{L}(M)_{d}$ being generated by the Lie monomials of length $d$. There is an obvious extension of the anchor $\mathcal{L}(M) \rightarrow \operatorname{Der}_{\mathbb{R}}(A)$. The free Lie-Rinehart algebra $\mathcal{R}(M)$, generated by $M$, is a filtered $A$-module, inductively defined as follows:

- $\mathcal{R}(M)_{\leq 1}=M$;

- for $n>1 \mathcal{R}(M)_{\leq d}$ is $\bigoplus_{1 \leq i \leq d} \mathcal{L}(M)_{i}$ modulo the relations

$$
[x, f y]-[f x, y]=x(f) y+y(f) x, \quad[x, q]=0,
$$

where $x, y \in \mathcal{L}(M), f \in A, q$ is in the kernel of

$$
\bigoplus_{1 \leq i \leq d-1} \mathcal{L}(M)_{i} \rightarrow \mathcal{R}(M)_{\leq d-1},
$$

and for any $x \in \mathcal{L}(M)$ we write $x(f)$ for the action of $x$ on $f$ through the anchor. It is easy to see that the kernel of $\mathcal{L}(M) \rightarrow \mathcal{R}(M)$ is a Lie ideal and hence $\mathcal{R}(M)$ inherits a Lie structure. It is also easy to check that the anchor $\mathcal{L}(M) \rightarrow \operatorname{Der}_{\mathbb{R}}(A)$ vanishes on the kernel of $\mathcal{L}(M) \rightarrow \mathcal{R}(M)$ and hence there is a well-defined action of $\mathcal{R}(M)$ on $A$. Finally, the action of $A$ on $\mathcal{R}(M)$ is given by

$$
f[x, y]=[f x, y]+y(f) x=[x, f y]-x(f) y .
$$

Applying the above construction to the set $\mathfrak{X}^{1}$ of vector fields on $\mathcal{M}$, we get a new Lie-Rinehart algebra $\mathcal{R}\left(\mathfrak{X}^{1}\right)$ which is the space of infinitesimal paths that do not contain any contractions of surfaces other than the degenerate ones (that is, we do have $[\alpha, \alpha]=0)$. The sheaf $\mathcal{R}\left(\mathfrak{X}^{1}\right)$ is locally free and hence we can form the linear bundle $\mathcal{R} T M$ which has $\mathcal{R}\left(\mathfrak{X}^{1}\right)$ as the set of sections. We would like to iterate this construction. That is, we would like to consider spaces of infinitesimal paths on the spaces of infinitesimal paths on $\mathcal{M}$, and so on. First we need to establish some of the functorial properties of $\mathcal{R}$. 
Proposition 3.1. Let $\mathcal{M}$ and $\mathcal{N}$ be two manifolds and let $F: \mathcal{M} \rightarrow \mathcal{N}$ be a smooth map that is an embedding locally (on $\mathcal{M})$. Then $F$ extends to a map of pairs

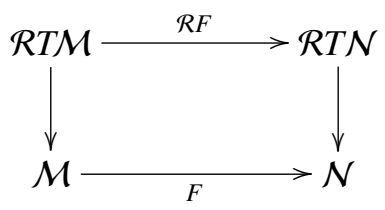

and this extension is functorial in $F$.

Proof. Since the construction of $\mathcal{R} T$ can be done locally (see [2]), we can (choosing local coordinates) assume that $\mathcal{M}=\mathbb{R}^{m}, \mathcal{N}=\mathbb{R}^{n}=\mathbb{R}^{m} \times \mathbb{R}^{k}$ and $F: \mathbb{R}^{m} \rightarrow \mathbb{R}^{m} \times \mathbb{R}^{k}$ is the inclusion of a coordinate subspace. Using the natural flat structure on $\mathbb{R}^{n}$, we have a map

$$
F_{*}: \mathfrak{X}^{1}\left(\mathbb{R}^{m}\right) \rightarrow \mathfrak{X}^{1}\left(\mathbb{R}^{n}\right)
$$

that is a morphism of Lie algebras. Consequently there is an induced morphism

$$
\mathcal{L}\left(F_{*}\right): \mathcal{L}\left(\mathfrak{X}^{1}\left(\mathbb{R}^{m}\right)\right) \rightarrow \mathcal{L}\left(\mathfrak{X}^{1}\left(\mathbb{R}^{n}\right)\right) .
$$

There is also a projection

$$
\mathbb{R}^{n}=\mathbb{R}^{m} \times \mathbb{R}^{k} \rightarrow \mathbb{R}^{m},
$$

and hence we have an inclusion of algebras of functions

$$
\mathfrak{X}^{0}\left(\mathbb{R}^{m}\right) \rightarrow \mathfrak{X}^{0}\left(\mathbb{R}^{n}\right) \text {. }
$$

Using this inclusion, it is easy to see that $\mathcal{L}\left(F_{*}\right)$ maps the kernel of the map $\mathcal{L}\left(\mathfrak{X}^{1}\left(\mathbb{R}^{m}\right)\right) \rightarrow \mathcal{R}\left(\mathfrak{X}^{1}\left(\mathbb{R}^{m}\right)\right)$ to the kernel of $\mathcal{L}\left(\mathfrak{X}^{1}\left(\mathbb{R}^{m}\right)\right) \rightarrow \mathcal{R}\left(\mathfrak{X}^{1}\left(\mathbb{R}^{m}\right)\right)$ and hence we have an $\mathbb{R}$-linear map

$$
\mathcal{R}\left(F_{*}\right): \mathcal{R}\left(\mathfrak{X}^{1}\left(\mathbb{R}^{m}\right)\right) \rightarrow \mathcal{R}\left(\mathfrak{X}^{1}\left(\mathbb{R}^{n}\right)\right) .
$$

This map is also $\mathfrak{X}^{0}\left(\mathbb{R}^{m}\right)$-linear, where we see $\mathcal{R}\left(\mathfrak{X}^{1}\left(\mathbb{R}^{n}\right)\right)$ as an $\mathfrak{X}^{0}\left(\mathbb{R}^{m}\right)$-module through (3.2). Now we compose $\mathcal{R}\left(F_{*}\right)$ with the projection

$$
\mathcal{R}\left(\mathfrak{X}^{1}\left(\mathbb{R}^{n}\right)\right) \rightarrow \mathcal{R}\left(\mathfrak{X}^{1}\left(\mathbb{R}^{n}\right)\right) \otimes_{\mathfrak{x}^{0}}\left(\mathbb{R}^{n}\right) \mathfrak{X}^{0}\left(\mathbb{R}^{m}\right),
$$

and obtain a morphism of bundles

$$
\mathcal{R} F: \mathcal{R} T M \rightarrow F^{*}(\mathcal{R} T \mathcal{N}) .
$$

We claim that $\mathcal{R} F$ is independent of the choice of local coordinates on $\mathcal{N}$. Indeed, a different choice produces different maps in (3.1) and (3.2), but they become the same when restricted to the image of $\mathcal{M}$ in $\mathcal{N}$. It is easy to check that this implies that the resulting $\mathcal{R} F$ s are equal.

Since there are two smooth maps $\mathcal{M} \rightarrow \mathcal{N} \rightarrow \mathcal{N}^{\prime}$, each of which is a local embedding, it is clear that we can locally present these maps as inclusions of coordinate subspaces $\mathbb{R}^{m} \rightarrow \mathbb{R}^{m+k} \rightarrow \mathbb{R}^{m+k+k^{\prime}}$. The corresponding choice of local coordinates implies functoriality. 
Now we would like to iterate the $\mathcal{R} T$ construction. In some of our arguments we shall assume that the manifolds in question are finite-dimensional, while $\mathcal{R} T \mathcal{M}$ is infinite-dimensional if the dimension of $\mathcal{M}$ is greater than 1 . However, since $\mathcal{R} T \mathcal{M}$ is filtered and each $\mathcal{R} T \mathcal{M}_{\leq d}$ is finite-dimensional, we can treat $\mathcal{R} T \mathcal{M}$ as if it is finitedimensional itself, so long as everything that we do occurs in some $\mathcal{R} T \mathcal{M}_{\leq d}$ for large enough $d$. We are interested, in particular, in tangent vectors to $\mathcal{R} T \mathcal{M}$ and we will always assume that each vector is tangent to some $\mathcal{R T M}_{\leq d}$. Therefore, so long as there are only finitely many tangents involved, there is a finite-dimensional manifold $\mathcal{R T M}_{\leq d}$ where these tangents live.

Let $\mathfrak{X}^{1}(\mathcal{R} T M)$ be the space of vector fields on $\mathcal{R} T \mathcal{M}$ such that for every field $\alpha$ there is a $d<\infty$ which satisfies the condition that $\alpha$ is tangent to $\mathcal{R} T \mathcal{M}_{\leq d}$. Applying $\mathcal{R}$ for each $d$ and using the functoriality of $\mathcal{R}$ with respect to local embeddings, we obtain a bundle $(\mathcal{R} T)^{2} \mathcal{M}$ of infinitesimal paths on $\mathcal{R} T \mathcal{M}$.

Iterating this procedure further, we get a sequence of (filtered infinite-dimensional) manifolds $\left\{(\mathcal{R} T)^{k} \mathcal{M}\right\}_{k \geq 1}$. However, this sequence is not the right substitute for the (semisimplicial) sequence $\left\{T^{k} \mathcal{M}\right\}_{k \geq 1}$ of iterated tangent bundles.

For $k \geq 2$ the manifold $(\mathcal{R} T)^{k} \mathcal{M}$ is too big. For example, there are loops in $(\mathcal{R} T)^{2} \mathcal{M}$ that are built out of vector fields which are tangent to the fibers of $\mathcal{R} T \mathcal{M} \rightarrow \mathcal{M}$. These fibers are linear spaces and have a natural flat connection. As far as paths and surfaces on $\mathcal{M}$ are concerned, we are only interested in flat vertical fields on $\mathcal{R} T \mathcal{M}$ and the corresponding loops have unique flat fillings.

All of this forces us to introduce a relative version of the free Lie-Rinehart algebra construction. Instead of anchored modules we have the following.

Definition 3.2. Let $A$ be a commutative $\mathbb{R}$-algebra and let $\left(\mathfrak{g} \stackrel{a}{\longrightarrow} \operatorname{Der}_{\mathbb{R}}(A)\right)$ be a LieRinehart algebra. An anchored $(\mathfrak{g}, A)$-module is an $A$-module $M$, together with A-maps

$$
\mathfrak{g} \stackrel{\iota}{\rightarrow} M \stackrel{b}{\longrightarrow} \operatorname{Der}_{\mathbb{R}}(A),
$$

such that $\iota$ is injective, $a=b \iota$ and there is a Lie module structure $\mathfrak{g} \otimes_{\mathbb{R}} M \rightarrow M$ such that

$$
\begin{gathered}
\gamma(f m)=\gamma(f) m+f \gamma(m), \quad(f \gamma)(m)=f \gamma(m)-m(f) \iota(\gamma), \\
b(\gamma(m))=[a(\gamma), b(m)] .
\end{gathered}
$$

An example of an anchored $(\mathfrak{g}, A)$-module is given by a morphism of Lie-Rinehart algebras $\mathfrak{g} \rightarrow \mathfrak{h}$ over $A$, where we take $\mathfrak{h}$ to be $M$. If we fix $\mathfrak{g}$ and $A$ we have a forgetful functor from the category of Lie-Rinehart algebras under $g$ to the category of anchored ( $\mathfrak{g}, A)$-modules. This functor has a left adjoint which we now describe.

Let $M$ be an anchored $(\mathfrak{g}, A)$-module. Recall that $\mathcal{L}(M)$ is the free Lie algebra over $\mathbb{R}$, generated by $M$. The action of $\mathfrak{g}$ on $M$ extends to an action on $\mathcal{L}(M)$, under the condition that

$$
\gamma([x, y])=[\gamma(x), y]+[x, \gamma(y)]
$$


We inductively define $\mathcal{R}(M, \mathfrak{g})$ as follows:

- $\quad \mathcal{R}(M, \mathfrak{g})_{\leq 1}=M$;

- $\quad$ if $d>1$, then $\mathcal{R}(M, \mathfrak{g})_{\leq d}$ is $\bigoplus_{1 \leq i \leq d} \mathcal{L}(M)_{i}$ modulo the relations

$$
\begin{gathered}
{[x, f y]-[f x, y]=x(f) y+y(f) x, \quad[x, q]=0,} \\
{[\iota(\gamma), x]=\gamma(x),}
\end{gathered}
$$

where $x, y \in \mathcal{L}(M), f \in A, \gamma \in \mathfrak{g}, q$ is in the kernel of

$$
\bigoplus_{1 \leq i \leq n-1} \mathcal{L}(M)_{i} \rightarrow \mathcal{R}(M)_{\leq n-1}
$$

and for any $x \in \mathcal{L}(M)$ we write $x(f)$ for the action of $x$ on $f$ through the anchor. Here we consider $\mathcal{L}(M)$ as an $\mathbb{R}$-space and take the quotient by the subspace generated by (3.5) and (3.6). From the construction it is clear that $\mathcal{R}(M, \mathfrak{g})$ is a Lie algebra over $\mathbb{R}$, inheriting the Lie structure from $\mathcal{L}(M)$. We claim that, in addition, $\mathcal{R}(M, \mathfrak{g})$ is an $A$-module and the projection $\mathcal{L}(M) \rightarrow \mathcal{R}(M, \mathfrak{g})$ factors through $\mathcal{R}(M)$. Notice that the action of $\mathfrak{g}$ on $\mathcal{L}(M)$ is compatible with (3.5), that is, the kernel of $\mathcal{L}(M) \rightarrow \mathcal{R}(M)$ is stable under the action of $\mathfrak{g}$. Therefore this action extends to $\mathcal{R}(M)$ and hence the relations (3.6) are well defined on $\mathcal{R}(M)$. This implies that $\mathcal{L}(M) \rightarrow \mathcal{R}(M, \mathfrak{g})$ factors through $\mathcal{R}(M)$.

There is an $A$-module structure on $\mathcal{R}(M)$, given by

$$
f[x, y]=[f x, y]+y(f) x=[x, f y]-x(f) y .
$$

We claim that the kernel of $\mathcal{R}(M) \rightarrow \mathcal{R}(M, \mathfrak{g})$ is an $A$-submodule. Indeed,

$$
f([\iota(\gamma), x]-\gamma(x))=[\iota(\gamma), f x]-\gamma(f x),
$$

for any $x \in \mathcal{L}(M), f \in A$, and $\gamma \in \mathfrak{g}$. Consequently $\mathcal{R}(M, \mathfrak{g})$ is an $A$-module. Finally, we note that $\mathcal{R}(M, \mathfrak{g})$ inherits an action on $A$ from $\mathcal{R}(M)$. This is rather obvious, since elements of the kernel of $\mathcal{R}(M) \rightarrow \mathcal{R}(M, \mathfrak{g})$ act trivially on $A$ (this is a consequence of relation (3.4)).

For us, the main applications of the relatively free Lie-Rinehart algebra construction are for locally trivial bundles, that is, smooth maps $F: \mathcal{M} \rightarrow \mathcal{N}$ such that $F$ is locally (on $\mathcal{M}$ ) a trivial bundle. Let $\mathfrak{X}^{1}(F)$ be the vector fields tangent to the fibers of $F$. Then we have the relatively free Lie-Rinehart algebra $\mathcal{R}\left(\mathfrak{X}^{1}(\mathcal{M}), \mathfrak{X}^{1}(F)\right.$ ) over $\mathfrak{X}^{0}(\mathcal{M})$ that defines the bundle $\mathcal{R} T_{\mathcal{N}} \mathcal{M}$ of $F$-horizontal infinitesimal paths on $\mathcal{M}$.

If we choose $F$ to be the unique map $\mathcal{M} \rightarrow \mathrm{pt}$, then we have $\mathcal{R} T_{\mathrm{pt}} \mathcal{M}=T \mathcal{M}$, the usual tangent bundle. If we choose $F$ to be the identity map $\mathcal{M}=\mathcal{M}$, then we have $R T_{\mathcal{M}} \mathcal{M}=\mathcal{R} T \mathcal{M}$, the space of infinitesimal paths from [2].

A nice property of $\mathcal{R} T_{\mathcal{N}} \mathcal{M}$ that will be used in the next section is that it is functorial in both arguments, as the following proposition shows. 
Proposition 3.3. Let $F_{1}: \mathcal{M}_{1} \rightarrow \mathcal{N}_{1}$ and $F_{2}: \mathcal{M}_{2} \rightarrow \mathcal{N}_{2}$ be two locally (on domains) trivial bundles. Suppose that we are given a commutative diagram of smooth maps

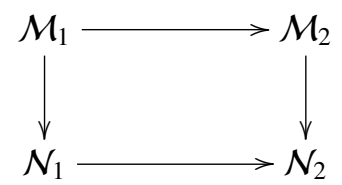

such that the horizontal arrows are also locally trivial bundles. Then we have a smooth map

$$
\mathcal{R} T_{\mathcal{N}_{1}} \mathcal{M}_{1} \rightarrow \mathcal{R} T_{\mathcal{N}_{2}} \mathcal{M}_{2}
$$

and (3.8) is functorial in (3.7).

Proof. First we prove functoriality in the second variable by considering the diagram.

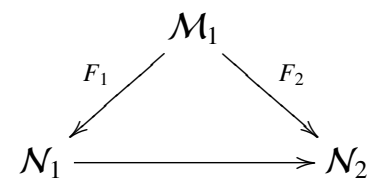

It is clear that $\mathfrak{X}^{1}\left(F_{1}\right)<\mathfrak{X}^{1}\left(F_{2}\right)<\mathfrak{X}^{1}\left(\mathcal{M}_{1}\right)$ as Lie algebras and therefore it is easy to check that there is a canonical surjective morphism of Lie-Rinehart algebras

$$
\mathcal{R}\left(\mathfrak{X}^{1}\left(\mathcal{M}_{1}\right), \mathfrak{X}^{1}\left(F_{1}\right)\right) \rightarrow \mathcal{R}\left(\mathfrak{X}^{1}\left(\mathcal{M}_{1}\right), \mathfrak{X}^{1}\left(F_{2}\right)\right)
$$

and hence a morphism of bundles $\mathcal{R} T_{\mathcal{N}_{1}} \mathcal{M}_{1} \rightarrow \mathcal{R} T_{\mathcal{N}_{2}} \mathcal{M}_{1}$ that is obviously functorial in (3.9).

Now we prove functoriality in the first variable. Choosing local coordinates, we can represent $F_{1}$ as the projection $\mathbb{R}^{k+n}=\mathbb{R}^{k} \times \mathbb{R}^{n} \rightarrow \mathbb{R}^{n}$. Then every element of $\mathcal{R}\left(\mathfrak{X}^{1}\left(\mathcal{M}_{1}\right), \mathfrak{X}^{1}\left(F_{1}\right)\right)$ can be written as follows:

$$
\sum_{i=1}^{\infty} f_{j_{1}, \ldots, j_{i}}\left[\partial_{j_{1}},\left[\cdots\left[\partial_{j_{i-1}}, \partial_{j_{i}}\right] \cdots\right]\right]
$$

where the sum is finite and $i>1$ implies that $f_{j_{1}, \ldots, j_{i}}=0$ if at least one of the $\partial_{j}$ is tangent to the fibers of $F_{1}$.

Consider the diagram.

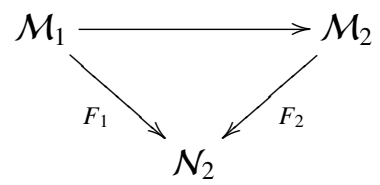


Since all maps are locally trivial bundles, we can choose local coordinates in a compatible way. That is, locally (3.10) becomes

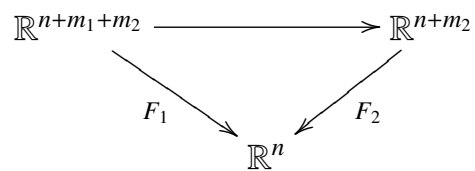

and it is clear how to define the map $\mathcal{R} T_{\mathcal{N}_{2}} \mathcal{M}_{1} \rightarrow \mathcal{R} T_{\mathcal{N}_{2}} \mathcal{M}_{2}$ locally. Functoriality and independence of the choice of coordinates are easy to check.

\section{The full infinitesimal groupoid}

In this section we use the relatively free Lie-Rinehart construction of the previous section to define the sequence $\left\{\mathbb{T}^{k} \mathcal{M}\right\}_{k \geq 0}$ of iterated bundles of spaces of infinitesimal paths on a manifold $\mathcal{M}$. In particular, we obtain a semisimplicial structure on $\left\{\mathbb{T}^{k} \mathcal{M}\right\}_{k \geq 1}$. For $k \geq 1$ we define $\mathbb{X}^{k}$ to be the set of sections $\mathcal{M} \rightarrow \mathbb{T}^{k} \mathcal{M}$ and define $\mathbb{X}^{0}:=\mathfrak{X}^{0}(\mathcal{M})$. We show how every $v \in \mathbb{X}^{k}$ for $k \geq 1$ can be decomposed into a sequence $\left\{\alpha_{\phi}\right\}$ with $\alpha_{\phi} \in \mathbb{X}^{1}$ and $\phi$ running over the nonempty subsets of $\{0, \ldots, k-1\}$. This allows us to define a rich algebraic structure on $\mathbb{X}^{*}:=\left\{\mathbb{X}^{k}\right\}_{k \geq 0}$ which we call the full infinitesimal groupoid of $\mathcal{M}$. Finally, we show that the nonlinear cohomology of $\mathbb{X}^{*}$ is the algebra of polyvector fields on $\mathcal{M}$.

Definition 4.1. Let $\mathcal{M}$ be a manifold. We define a sequence of (generally nonlinear) locally trivial bundles $\left\{\pi_{k}: \mathbb{T}^{k} \mathcal{M} \rightarrow \mathcal{M}\right\}_{k \geq 0}$ as follows. We set $\mathbb{T}^{0} \mathcal{M}:=\mathcal{M}$. If we have defined $\pi_{k}: \mathbb{T}^{k} \mathcal{M} \rightarrow \mathcal{M}$, then

$$
\mathbb{T}^{k+1} \mathcal{M}:=\mathcal{R} T_{\mathcal{M}}\left(\mathbb{T}^{k} \mathcal{M}\right)
$$

and the projection $\pi_{k+1}: \mathbb{T}^{k+1} \mathcal{M} \rightarrow \mathcal{M}$ is obvious.

Notice that, just like $\left\{T^{k} \mathcal{M}\right\}_{k \geq 1}$, the sequence $\left\{\mathbb{T}^{k} \mathcal{M}\right\}_{k \geq 1}$ has a semisimplicial structure, that is, for each $k \geq 1$ there are $k$ projections

$$
\pi_{k, i}: \mathbb{T}^{k} \mathcal{M} \rightarrow \mathbb{T}^{k-1} \mathcal{M}, \quad 0 \leq i \leq k-1 .
$$

When $i=0$ note that $\pi_{k, 0}$ is the projection of the bundle $\mathcal{R} T_{\mathcal{M}}\left(\mathbb{T}^{k-1} \mathcal{M}\right)$ on $\mathbb{T}^{k-1} \mathcal{M}$. When $i>0$ the operator $\pi_{k, i}$ is obtained from $\pi_{k-i, 0}$ by functoriality of $\mathcal{R} T$. Moreover, each $\pi_{k, i}$ is a linear bundle. This is a general fact: consider a morphism of locally trivial bundles

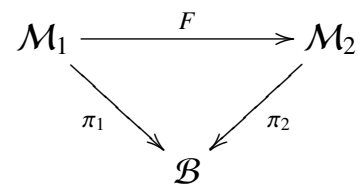


such that $F$ is a linear bundle. By the functoriality of $\mathcal{R} T$ we have the diagram

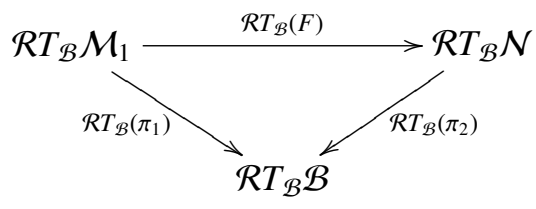

It is easy to see that fibers of $\mathcal{R} T_{\mathcal{B}}\left(\pi_{1}\right)$ are the tangent bundles to the fibers of $\pi_{1}$, and similarly for $\pi_{2}$. Therefore fibers of $\mathcal{R} T_{\mathcal{B}}(F)$ are the same as fibers of $T(F)$. It is well known (see, for example, [4]) that $T(F)$ is a linear bundle whenever $F$ is.

Let $\mathbb{X}^{0}:=\mathfrak{X}^{0}(\mathcal{M})$ and let $\mathbb{X}^{k}$ be the set of sections of $\mathbb{T}^{k} \mathcal{M} \rightarrow \mathcal{M}$. Just as with $\mathfrak{X}^{k}$, using the functoriality of $\mathcal{R} T$ with respect to local embeddings (see Proposition 3.1), we obtain a decomposition of each $v \in \mathbb{X}^{k}$ into a set $\left\{\alpha_{\phi}\right\}$ where each $\alpha \in \mathbb{X}^{1}$ and $\phi$ runs over all nonempty subsets of $\{1, \ldots, k\}$. As with $\mathfrak{X}^{k}$, for $v \in \mathbb{X}^{k}$ the decomposition into $\left\{\alpha_{\phi}\right\}$ depends on the order placed on the set of projections of $v$ on $\mathbb{X}^{1}$. We also have an action of the symmetric group $\mathbb{S}_{k}$ that is given as in (2.4) here, but with the Lie bracket substituted by the free bracket $\llbracket-,-\rrbracket$ on $\mathbb{X}^{1}=\mathcal{R} T\left(\mathfrak{X}^{1}\right)$. It is clear how to extend additions, strong differences, the cup product and the composition product from $\left\{\mathfrak{X}^{k}\right\}$ to $\left\{\mathbb{X}^{k}\right\}$. We will freely use the notation of Section 2. We will call $\mathbb{X}^{*}:=\left\{\mathbb{X}^{k}\right\}$ together with these (and other) operations the full infinitesimal groupoid of $\mathcal{M}$.

In addition to the operations listed above, $\mathbb{X}^{*}$ has homotopy operations, defined to be maps

$$
\left\{\mathbb{X}^{k} \stackrel{h_{i j}^{k}}{\longrightarrow} \mathbb{X}^{k-1}\right\}_{0 \leq i<j \leq k-1}
$$

for each $k \geq 2$. To define the homotopy operations we notice that $\mathbb{X}^{1}$ actually has two Lie structures. They come from two Lie structures on $\mathcal{L}\left(\mathfrak{X}^{1}\right)$. The first one is the free bracket $\llbracket-,-\rrbracket$ given by $\mathcal{L}$, and the other is the Lie bracket $[-,-]$ on $\mathfrak{X}^{1}$, extended to $\mathcal{L}\left(\mathfrak{X}^{1}\right)$ by the requirement that

$$
[x, \llbracket y, z \rrbracket]=\llbracket[x, y], z \rrbracket+\llbracket y,[x, z] \rrbracket .
$$

It is easy to check that the kernel of $\mathcal{L}\left(\mathfrak{X}^{1}\right) \rightarrow \mathcal{R}\left(\mathfrak{X}^{1}\right)$ is also a Lie ideal for $[-,-]$ and hence $\mathbb{X}^{1}$ inherits $[-,-]$.

Now, having an additional bracket on $\mathbb{X}^{1}$, we have an additional action of $\mathbb{S}_{k}$ on $\mathbb{X}^{k}$ which can be written in terms of $\left\{\alpha_{\phi}\right\}$ and $\alpha_{\phi} \in \mathbb{X}^{1}$. That is, we apply the same formula (2.4), but we use [-, - ] instead of the free bracket. For $k \geq 2$ let $v \in \mathbb{X}^{k}$ and let $\sigma_{i j} \in \mathbb{S}_{k}$ be the swapping of $i$ and $j$. Let $\mu \in \mathbb{X}^{k-2}$ be the projection of $v$ on the $\phi$-face, where

$$
\phi=\{0, \ldots, \widehat{i}, \ldots, \widehat{j}, \ldots, k-1\}
$$

(if $k=2$, then $\mu$ is just $\mathcal{M}$ itself). Clearly $\mu$ is also projection of $\sigma_{i j}(v)$ and $\sigma_{i j}^{\prime}(v)$ where $\sigma_{i j}$ acts using $\mathbb{[}-,-\rrbracket$ and $\sigma_{i j}^{\prime}$ acts using $[-,-]$.

As 2-vector fields on the image of $\mu$ in $\mathbb{T}^{k-2} \mathcal{M}$, the vector fields $\sigma_{i j}(v)$ and $\sigma_{i j}^{\prime}(v)$ have the same boundaries and we can take their strong difference. It is the element $h_{i j}^{k}(v)$ of $\mathbb{X}^{k-1}$. A straightforward but long computation shows that, modulo 
the homotopies $h_{* *}^{<k}, h_{i j}^{k}$ is well defined with respect to the actions of symmetric groups. That is, for any $\sigma \in \mathbb{S}_{k}$ there is $\tau \in \mathbb{S}_{k-1}$ such that

$$
h_{\sigma(i) \sigma(j)}^{k}(\sigma v) \sim \tau\left(h_{i j}^{k}(v)\right) .
$$

The equivalence relation on $\mathbb{X}^{k-1}$ defined by $h_{i j}^{k}(v)$ is the following: for $\xi, \xi^{\prime} \in \mathbb{X}^{k-1}$ we say that $\xi \sim \xi^{\prime}$ if $\xi-_{\phi} \xi^{\prime}=h_{i j}^{k}(v)$. Consequently, it is natural to say that $v \in \mathbb{X}^{k}$ defines trivial homotopies if $\sigma_{i j}(v)=\sigma_{i j}^{\prime}(v)$ for any $0 \leq i<j \leq k-1$, that is, if $h_{i j}^{k}(v)$ consists of trivial vectors on the $\phi$-face of $v$.

What do we get if we take the subset of $\mathbb{X}^{*}$, consisting of elements that define trivial homotopies, and divide it by the homotopy equivalence? We denote the result by $\mathcal{H}\left(\mathbb{X}^{*}\right)$ and call it the cohomology of $\mathbb{X}^{*}$.

First of all, it is clear that any $v \in \mathbb{X}^{k}$ is equivalent to some $v^{\prime} \in \mathfrak{X}^{k}$ and no two $v^{\prime}, v^{\prime \prime} \in \mathfrak{X}^{k}$ are equivalent. Secondly, any $\left\{\alpha_{\phi}\right\}=v \in \mathfrak{X}^{k}$ defines trivial homotopies if and only if for any $\phi, \psi \subseteq\{0, \ldots, k-1\}$ such that $\phi \cap \psi=\emptyset$ we have that either $\alpha_{\phi}=\alpha_{\psi}$ or at least one of $\alpha_{\phi}, \alpha_{\psi}$ is 0 . This is just the condition for $\llbracket \alpha_{\phi}, \alpha_{\psi} \rrbracket=0$. There are quite a large number of such $v \mathrm{~s}$, but not so many if we discard degenerate $k$-submanifolds and quotient out by reparametrizations. Note that by taking the canonical zero section we can consider any $\alpha \in \mathfrak{X}^{1}$ to be a section of $T^{2} \mathcal{M}$, that is, we take $\alpha \cup 0$ which is obviously a degenerate 2-vector field. Also, $\alpha \cup \alpha$ is degenerate since it is the jet of a one-dimensional submanifold. Together we have

$$
\alpha \sim \alpha \cup 0 \sim \alpha \cup \alpha \sim 0 \cup \alpha .
$$

In addition, for $k \geq 2$ we have reparametrizations of $k$-vectors, that is, maps $\mathcal{W}_{k} \rightarrow$ $\mathcal{W}_{k}$, that have 1 as their Jacobian. Discarding degenerate fields implies that any $v \in \mathfrak{X}^{*}$ that defines trivial homotopies is equivalent to some $v^{\prime}=\left\{\alpha_{\phi}^{\prime}\right\}$ which satisfies the condition that $\alpha_{\phi}^{\prime}=0$, unless $\phi=\{0, \ldots, i\}$ for some $i \leq k-1$. Quotienting out by reparametrizations means that $v^{\prime}$ is linear over $\mathfrak{X}^{0}$ in each one of $\alpha_{\phi}^{\prime}$ s and, discarding degenerate fields again, we get that $v^{\prime}$ is an element of some alternating power of $\mathfrak{X}^{1}$ over $\mathfrak{X}^{0}$. That is, $\mathcal{H}\left(\mathbb{X}^{*}\right)$ is the set of decomposable elements of $\wedge^{*} \mathfrak{X}^{1}$. Taking the $\mathfrak{X}^{0}$-module generated by $\mathcal{H}\left(\mathbb{X}^{*}\right)$, we get all of $\wedge^{*} \mathfrak{X}^{1}$.

Finally, we discuss algebraic operations on $\mathcal{H}\left(\mathbb{X}^{*}\right)$. On $\mathbb{X}^{*}$ we have some additions, the cup product and the composition product. The additions translate to the addition on $\wedge^{*} \mathfrak{X}^{1}$ and the cup product becomes the wedge product. It is not so simple for the composition product. Recall how one defines the Lie derivative of a vector field $\alpha$ along another vector field $\beta$. One takes the composition product $\alpha \times \beta \in \mathfrak{X}^{2}$, uses trivialization of $T M$ over the integral curves of $\beta$ to find the projection of $\alpha \times \beta$ on the fibers of $T M$ and then uses the linear structure on these fibers to identify the tangents to the fibers with their points. The resulting section of $T M$ is $[\beta, \alpha]$. In terms of higher categories this is what is called $a$ thin structure, that is, $\alpha \times \beta$ is not the composition, but one of its faces is. Since $\mathcal{H}\left(\mathbb{X}^{*}\right)$ is not additive, but multi-linear, we need not one face but many and the resulting operation is the Schouten bracket. The composition product is also a thin structure on all of $\mathbb{X}^{*}$, but its algebra of faces is beyond the scope of this paper. 
We would like to stress that $\wedge^{*} \mathfrak{X}^{1}$, obtained as above from $\mathcal{H}\left(\mathbb{X}^{*}\right)$, is not a Gerstenhaber algebra. Elements of $\wedge^{k} \mathfrak{X}^{1}$ represent $k$-morphisms and hence have degree $-k+1$, if we use cohomological notation. Therefore, while the bracket is of degree zero, the cup product is of degree -1 .

\section{References}

[1] W. Bertram, 'Differential geometry over general base fields and rings', Mem. Amer. Math. Soc. 192(900) (2008).

[2] M. Kapranov, 'Free Lie algebroids and the space of paths', Selecta Math. 13 (2007), 277-319.

[3] A. Kock and R. Lavendhomme, 'Strong infinitesimal linearity, with applications to strong difference and affine connections', Cah. Topol. Géom. Différ. 25 (1984), 311-324.

[4] K. C. H. Mackenzie, General Theory of Lie Groupoids and Lie Algebroids, London Mathematical Society Lecture Note Series, 213 (Cambridge University Press, Cambridge, 2005).

[5] I. Moerdijk and G. E. Reyes, Models for Smooth Infinitesimal Analysis (Springer, Berlin, 1991).

[6] J. E. White, The Method of Iterated Tangents with Applications in Local Riemannian Geometry, Monographs and Studies in Mathematics, 13 (Pitman, Boston, 1982).

DENNIS BORISOV, Max Planck Institute for Mathematics, PO Box 7280,

53072 Bonn, Germany

e-mail: dennis.borisov@gmail.com 